University of Nebraska - Lincoln

DigitalCommons@University of Nebraska - Lincoln

Robert Streubel Papers

Research Papers in Physics and Astronomy

$5-22-2012$

\title{
Magnetic vortices on closely packed spherically curved surfaces
}

\author{
Robert Streubel \\ Leibniz-Institut für Festkörper- und Werkstoffforschung Dresden, streubel@unl.edu \\ Denys Makarov \\ Leibniz-Institut für Festkörper- und Werkstoffforschung Dresden, d.makarov@ifw-dresden.de \\ Florian Kronast \\ Helmholtz-Zentrum Berlin für Materialien und Energie (HZB) \\ Volodymyr Kravchuk \\ Bogolyubov Institute for Theoretical Physics Nasu \\ Manfred Albrecht \\ Technische Universität Chemnitz
}

See next page for additional authors

Follow this and additional works at: https://digitalcommons.unl.edu/physicsstreubel

Part of the Atomic, Molecular and Optical Physics Commons, Condensed Matter Physics Commons, and the Other Physics Commons

Streubel, Robert; Makarov, Denys; Kronast, Florian; Kravchuk, Volodymyr; Albrecht, Manfred; and Schmidt, Oliver G., "Magnetic vortices on closely packed spherically curved surfaces" (2012). Robert Streubel Papers. 23.

https://digitalcommons.unl.edu/physicsstreubel/23

This Article is brought to you for free and open access by the Research Papers in Physics and Astronomy at DigitalCommons@University of Nebraska - Lincoln. It has been accepted for inclusion in Robert Streubel Papers by an authorized administrator of DigitalCommons@University of Nebraska - Lincoln. 


\section{Authors}

Robert Streubel, Denys Makarov, Florian Kronast, Volodymyr Kravchuk, Manfred Albrecht, and Oliver G.

Schmidt 


\title{
Magnetic vortices on closely packed spherically curved surfaces
}

\author{
Robert Streubel,,${ }^{1,2}$ Denys Makarov,,${ }^{1, *}$ Florian Kronast,${ }^{3}$ Volodymyr Kravchuk, ${ }^{4}$ Manfred Albrecht,${ }^{5}$ and Oliver G. Schmidt ${ }^{1,2}$ \\ ${ }^{1}$ Institute for Integrative Nanosciences, IFW Dresden, 01069 Dresden, Germany \\ ${ }^{2}$ Material Systems for Nanoelectronics, Chemnitz University of Technology, 09107 Chemnitz, Germany \\ ${ }^{3}$ Helmholtz-Zentrum Berlin für Materialien und Energie GmbH, 12489 Berlin, Germany \\ ${ }^{4}$ Bogolyubov Institute for Theoretical Physics, 03143 Kiev, Ukraine \\ ${ }^{5}$ Institute of Physics, Chemnitz University of Technology, 09107 Chemnitz, Germany \\ (Received 20 January 2012; revised manuscript received 26 April 2012; published 22 May 2012)
}

\begin{abstract}
We investigate the change of magnetic vortex states driven by curvature. The equilibrium state and magnetization reversal of soft magnetic permalloy ( $\mathrm{Py}, \mathrm{Ni}_{80} \mathrm{Fe}_{20}$ ) caps on self-assembled spherical particles with diameters of 100,330, and $800 \mathrm{~nm}$ are investigated, revealing the vortex ground state for individual caps and closely packed cap arrays. The magnetic coupling between vortices is substantially reduced due to the shape of the cap as apparent in a much weaker dependence of the magnetization reversal process on the separation distance. Interestingly, the remaining coupling is still sufficiently large to introduce chirality frustrated vortex states in closely packed cap arrays.
\end{abstract}

DOI: 10.1103/PhysRevB.85.174429

PACS number(s): 75.70.Kw, 75.60.Jk, 75.75.Fk, 81.16.Dn

Minimizing the size of soft-magnetic materials by applying microfabrication techniques yields to topologically stable objects like vortices. ${ }^{1}$ Magnetic vortices are characterized by the sense of the in-plane curling direction (clockwise or counterclockwise) of the magnetization (chirality) and the normal magnetization component in the center (polarity). ${ }^{2}$ This configuration minimizes the stray field and exhibits high stability with respect to temperature and magnetic field. Both vortex core motion ${ }^{3}$ and vortex switching by means of ac-magnetic fields ${ }^{4-6}$ and spin-polarized electric current ${ }^{7}$ were studied in planar structures experimentally and theoretically. ${ }^{8}$ Besides the aspect of fundamental investigation, vortices are also considered as prospective random access memory devices combining advantages of high-density storage and low-energy consumption. ${ }^{9}$ Although individual vortices are energetically preferred in patterned films, ${ }^{1}$ their arrangement in arrays of small periods leads to a significantly increased energy barrier for vortex nucleation due to magnetostatic coupling. ${ }^{10}$ This can even suppress the vortex remanent state. Therefore the coupling has been intentionally avoided by considering a distance between adjacent disks similar to the disk diameter. However, the magnetostatic coupling and thus the suppression of the vortex remanent state can be reduced by inserting a thickness gradient at the edge of the planar disk. ${ }^{11}$ An elegant way to provide a thickness gradient and to alter the magnetic interaction at the nanoscale simultaneously is to introduce a curvature. Previously, the substantial impact of a curvature on magnetic properties and magnetization reversal of thin hard magnetic films with out-of-plane easy axis ${ }^{12-14}$ was demonstrated. Very recently, theoretical calculations suggested that in thin spherical nanoshells a polarity-chirality coupling occurs for topological reasons. ${ }^{15}$ In this respect, curved magnetic films may exhibit intrinsically the possibility to switch the chirality by reversing the polarity or vice versa.

In this paper, we show that curvature modifies both stability of magnetic vortex states and coupling between adjacent vortices in permalloy caps on spherical particles, allowing us to fabricate closely packed vortex arrays with particle size down to $330 \mathrm{~nm}$. For smaller sizes, the vortex state is only favored for individual isolated caps. For an in-depth understanding of the magnetization process, the magnetic states were probed by means of x-ray magnetic circular dichroism photoemission electron microscopy (XMCD-PEEM) at BESSY II (beamline UE49-PGM, Helmholtz-Zentrum Berlin) while exposing the sample to an in-plane magnetic field. The vortices exhibit a significantly weaker dependence of the vortex nucleation and annihilation fields on the intercap distance compared with their planar counterparts due to a curvature-driven thickness variation that minimizes the magnetic stray field of the cap. A comparison of vortex nucleation and annihilation field of closely packed caps with individual disks reveals five to ten times smaller values. The corresponding value for individual disks and closely packed arrays of disks is $>25 .{ }^{10}$ Due to the reduced magnetostatic intercap interaction, we get insight into the coupling between vortices in closely packed cap arrays. In contrast to commonly considered spin frustrated systems, ${ }^{16,17}$ magnetic vortices in hemispherical closely packed cap arrays show chirality frustrated states that have not yet been reported in literature.

We report on magnetic equilibrium states and magnetization reversal of 20- and 40-nm-thick Py caps on selfassembled arrays of spherical $\mathrm{SiO}_{2}$ particles with diameters of $(100 \pm 15),(330 \pm 33)$, and $(800 \pm 40) \mathrm{nm}$ (Bangs Laboratories, Inc.). The silica particle arrays are fabricated by using the drop-cast approach. Throughout this paper, the notation $\operatorname{Py}(t / d)$ is used to refer to Py caps with thickness $t$ and diameter $d$ in $\mathrm{nm}$. The Py films as well as Ta buffer $(5 \mathrm{~nm})$ and capping $(2 \mathrm{~nm})$ layers were deposited by dc-magnetron sputtering at room temperature (Ar pressure: $10^{-3}$ mbar). A series of micromagnetic simulations was performed by OOMMF software ${ }^{18}$ that reveals the vortex ground state for individual magnetic Py caps. Scanning electron microscopy (SEM) topography analysis shows no overlap between adjacent caps suggesting a negligible magnetic intercap exchange interaction. Thus the magnetostatic coupling represents the major contribution in closely packed cap arrays. 

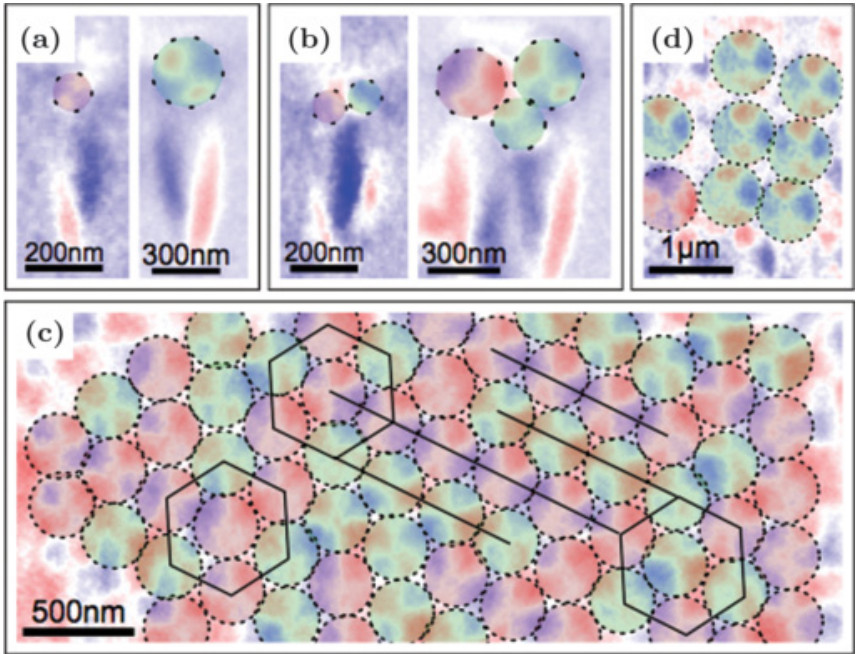

FIG. 1. (Color online) Magnetic equilibrium states of individual (a) and clusters (b) of hemispherical 20-nm-thick Py caps with diameters of 100 and $330 \mathrm{~nm}$ (indicated by dashed circles). Blue and red colors refer to in-plane magnetization components pointing up and down, respectively. Positive and negative chirality are indicated by red- and green-shaded circles, respectively. Panels (c) and (d) show equilibrium states of cap arrays with diameters of 330 and $800 \mathrm{~nm}$, respectively. Chirality frustrated vortex states form patterns with one-dimensional alternation (straight lines) or threefold symmetry (hexagon).

The visualization of magnetic states was carried out by XMCD-PEEM. In the used set-up, X-ray radiation hits the sample under an angle of $74^{\circ}$ with respect to the surface normal, which results for curved surfaces in an illumination of one side of the cap. The magnetic contrast originating from the opposite side of the cap is caused by transmitted $\mathrm{x}$-ray radiation and therefore inverted. ${ }^{19,20}$ Due to this geometry, the vortex state on spherical particles appears as characteristic quadrupolelike magnetic pattern (Fig. 1). For isolated spheres, the projection of the object's shadow onto the substrate causes a strong magnetic contrast. This feature, called "tail contrast," is of big advantage as it increases spatial resolution and allows us to observe vortices in caps with a diameter of $100 \mathrm{~nm}$.

The imaging of individual caps [Fig. 1(a)] and small clusters [Fig. 1(b)] of $\mathrm{Py}(20 / 100)$ and $\mathrm{Py}(20 / 330)$ caps (indicated by dashed circles) reveals vortex as equilibrium and remanent state after saturation. ${ }^{11,21}$ When two caps $(100$ or $330 \mathrm{~nm})$ are brought together, the magnetostatic interaction results in a chirality alternation [Fig. 1(b), left], as can be identified by following the tail contrast. Positive and negative chirality are indicated by red- and green-shaded regions, respectively. Adding a third cap to the arrangement results in the phenomenon of magnetic frustration [Fig. 1(b), right]. However, in contrast to classical frustration of magnetic moments, magnetic vortices in hemispherical closely packed cap arrays with diameters of $330 \mathrm{~nm}$ show chirality frustrated states [Fig. 1(c)]. As the name suggests, the vortices are frustrated by the sense of spin curling. This configuration is stabilized at remanence after saturation and is therefore nucleation driven. An applied magnetic field introduces a preferred magnetization orientation parallel to the field direction even if
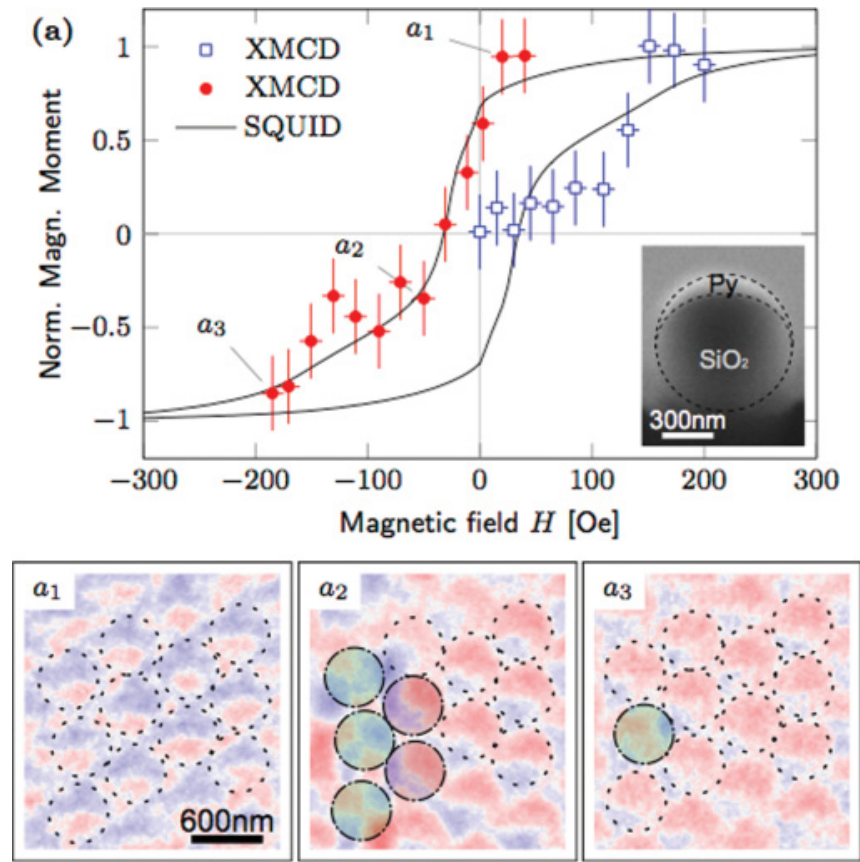

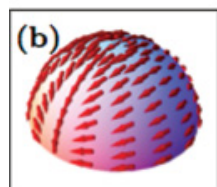

$H \approx 100 \mathrm{Oe}$

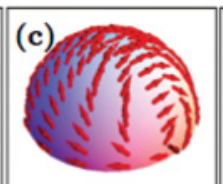

$\approx 0 \mathrm{Oe}$

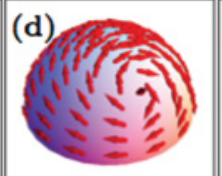

$0 \doteqdot-50 \mathrm{Oe}$

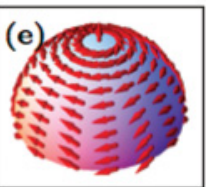

$\approx-50 \mathrm{Oe}$
FIG. 2. (Color online) (a) Magnetization reversal of an array consisting of $\mathrm{Py}(20 / 330)$ caps (indicated by dashed circles). The inset displays the cross section of a Py(40/800) cap. The images $a_{1}, a_{2}$, and $a_{3}$ illustrate remanent, transition, and saturation state, respectively. Caps that exhibit vortex states are indicated by $\cdot-$. The XMCD hysteresis curves are averaged over the upper side of these vortices. The integral hysteresis loop is plotted as reference (solid). Calculated transition states for decreasing field: (b) onion state; (c) $C$ state with vortex core at the edge; (d) shifted vortex; and (e) centered vortex state. The magnetic field values in panels $(b-d)$ are estimations only.

the field value approaches zero. Vortices nucleate pairwise with opposite chirality at the edge of caps perpendicular to the external magnetic field. Besides the one-dimensional chirality alternation, threefold symmetry arrangements also occur due to a thermally activated nucleation. The increase of the cap diameter to $800 \mathrm{~nm}$ prevents any correlation between chiralities of neighboring vortices [Fig. 1(d)], demonstrating the possibility of self-assembled closely packed vortex arrays.

For a detailed analysis of the magnetization reversal process of the cap array shown in Fig. 1(c), the magnetic states were probed while ramping an in-plane magnetic field. The orientation of the external field is along the column of caps in Fig. 2 (from top to bottom). The magnetic virgin curve is determined by numerical postprocessing of the XMCD-PEEM contrast on individual cap structures [Fig. 2(a)]. The error bars in Fig. 2(a) account for the statistical averaging over an ensemble of caps showing the vortex state. The observed transition at $(145 \pm 20)$ Oe matches very well with the vortex annihilation field 
derived from the local maximum of the susceptibility of the integral hysteresis loop of $(151 \pm 10)$ Oe that were obtained by superconducting quantum interference device-vibrating sample magnetometry (SQUID-VSM) measurements. In both disks and caps, stray fields originate from magnetic volume divergences and surface charges that are localized in the volume and at the edge of the circular pattern, respectively. For caps, the contribution of edge charges is negligible due to the continuously decreasing thickness. Hence smaller magnetic fields with respect to planar counterparts are required to annihilate the vortex in a cap. The magnetostatic coupling that suppresses the vortex nucleation for closely packed planar disks is much weaker in caps for the same reason.

After saturation the magnetic field was swept to monitor the magnetization reversal process [Fig. 2(a), red symbols]. The image sequence $\left(a_{1}, a_{2}, a_{3}\right)$ shows the evolution of the magnetic pattern from a dipolelike contrast in a single cap into a coexistence of dipole- and quadrupolelike contrast distributed over the cap array. The latter contrast is assigned to vortex indicated by - - and color shaded. Comparing the chirality of the observed vortices reveals a chirality frustrated state with a one-dimensional alternation. The dipolelike contrast is characteristic for a uniform state. Both hysteresis curve and XMCD-PEEM patterns can be understood by means of micromagnetic simulations. The calculated magnetic states are depicted in Figs. 2(b)-2(e) for decreasing magnetic field when coming from positive saturation. According to simulations the slow decrease of the magnetic signal at positive field corresponds to the relaxation of the magnetization into the surface plane forming the onion state [Fig. 2(b)]. As a next step, the irreversible magnetic transition is initiated by the transformation of the onion into the metastable $C$ state, apparent in the hysteresis curve by a constant permeability. With decreasing field the vortex core nucleates at the edge of the cap [Fig. 2(c)] in order to minimize the stray field. As the external magnetic field becomes more negative, the core is shifted to the center [Figs. 2(d) and 2(e)]. For even more negative fields, the core is displaced to the opposite edge with respect to the nucleation site and eventually expelled from the cap.

Closely packed arrays of caps with either larger thickness or larger diameter exhibit the vortex remanent state [Fig. 3(a)] due to an increased contribution of volume divergences in the onion state. Accordingly, vortex annihilation and nucleation field for $\mathrm{Py}(40 / 330)$ cap arrays are at room temperature $(-204 \pm 10)$ Oe and $(13 \pm 5)$ Oe, respectively. These values are substantially smaller (15-30\%) compared with individual planar disks ${ }^{22}$ (Table I). However, these fields are at least three times larger than those of closely packed arrays of planar disks ${ }^{10}$ allowing a vortex remanent state.

The temperature dependence of vortex annihilation and nucleation field for $\mathrm{Py}(40 / 330)$ cap arrays is shown in Figs. 3(b) and 3(c), respectively. For high temperatures, the slope that can be fitted by $H_{a n / n}(T)=H_{a n / n}(0)(1+$ $\alpha_{a n / n} T^{3 / 2}$ ) originates from the temperature dependence of the saturation magnetization due to spin-wave excitation (Bloch's law). The fitting parameters $\alpha_{n / a n}$ shown in Table I are twice the value of individual planar disks. ${ }^{22}$ The saturation magnetization measured at 300 Oe as a function of the temperature is fitted by $M_{S}(T)=M_{S}(0)(1-$
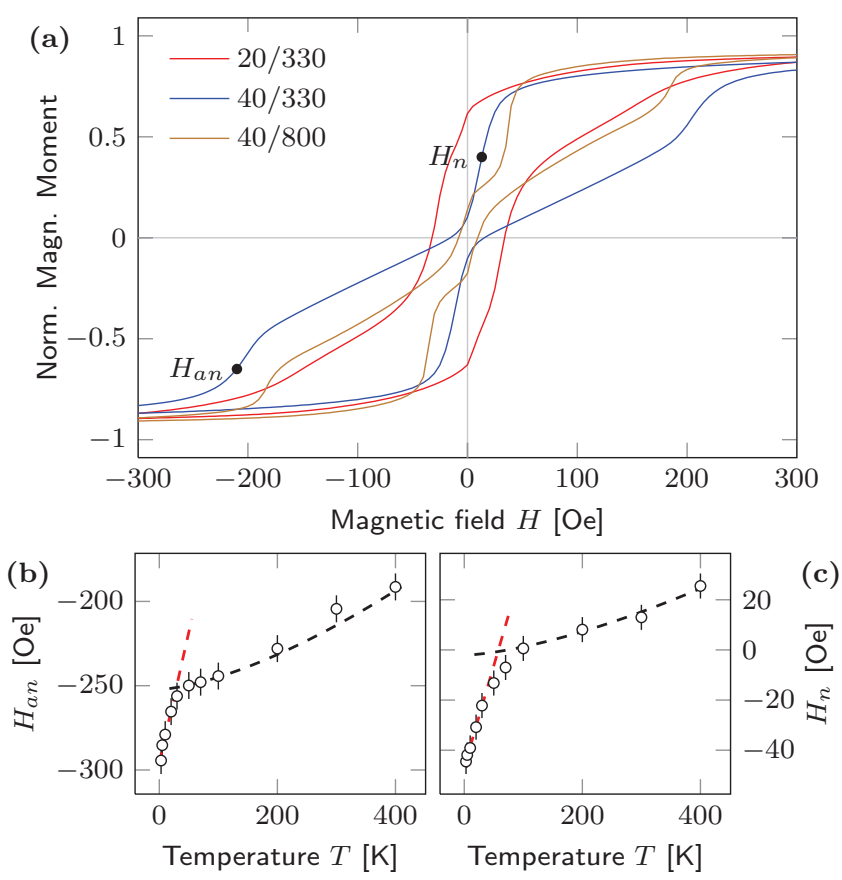

FIG. 3. (Color online) (a) Integral magnetic hysteresis loops of Py caps with thickness/diameter. Panels (b) and (c) illustrate the temperature dependence of vortex annihilation $\left(H_{a n}\right)$ and nucleation field $\left(H_{n}\right)$ of $\mathrm{Py}(40 / 330)$ caps taken at the local maximum of the susceptibility. The data (dashed lines) are fitted to the functions mentioned in the text.

$c_{1} T^{3 / 2}-c_{2} T^{2}$ ) with $c_{1}=3.65 \times 10^{-6} \mathrm{~K}^{-3 / 2}$ and $c_{2}=1.41 \times$ $10^{-7} \mathrm{~K}^{-2}$. Such an additional quadratic term was reported for nanoparticles, ${ }^{23}$ whose size reduces the $k$ space of spin waves significantly. In the case of magnetic caps, the thickness gradient causes a similar confinement. However, the small quadratic contribution was neglected for fitting $H_{a n / n}$.

For low temperatures $(<50 \mathrm{~K})$, the thermal activation of the magnetic transitions between onion and vortex state becomes the leading mechanism. Assuming a weak pinning and constant saturation magnetization, both vortex nucleation and annihilation field are fitted linearly by $H_{a n / n}(T)=H_{a n / n}(0)(1+$ $\left.\beta_{a n / n} T\right)$ with $\beta_{a n / n}=k_{B} / \Delta E_{a n / n} \ln \frac{f_{0} \tau}{\ln 2} \cdot{ }^{24} H_{a n / n}(0)$ is the field where the transition occurs without thermal activation; $\Delta E_{a n / n}, f_{0}$, and $\tau$ are the energy barrier for vortex annihilation/nucleation, the attempt frequency of relaxation, and the measurement time, respectively. Since $f_{0}$ is of the order $10^{10}-10^{12} \mathrm{~Hz},{ }^{25}$ the logarithm term contributes by a constant factor of $\approx 25$. The corresponding coefficients $\beta_{a n / n}$ are shown in Table I, with values justifying the assumption of weak pinning. The value of $\beta_{a n}$ for $\mathrm{Py}(20 / 330)$ cap arrays is much smaller due to the partial onion transition discussed above. In this approach, the energy barriers for vortex annihilation and nucleation in closely packed $\mathrm{Py}(40 / 330)$ caps are $(431 \pm 34)$ and $(127 \pm 10) \mathrm{meV}$, respectively.

In conclusion, we have presented experimental results on magnetic equilibrium states and magnetization reversal in 20- and 40-nm-thick Py caps on self-assembled spherical particles with diameters of 100,330 , and $800 \mathrm{~nm}$. The curvature-driven thickness gradient ensures the vortex ground state for both individual and closely packed caps due to 
TABLE I. Fitting parameters for temperature-dependent vortex nucleation $\left(H_{n}\right)$ and annihilation fields $\left(H_{a n}\right)$ that are described by $H_{a n / n}(T)=H_{a n / n}(0)\left(1-\alpha_{a n / n} T^{3 / 2}\right)(T>100 \mathrm{~K})$ and $H_{a n / n}(T)=H_{a n / n}(0)\left(1+\beta_{a n / n} T\right)(T<50 \mathrm{~K})$. The room-temperature values $H_{a n / n}^{r t}$ are also listed. For comparison, the fitting parameters for planar disks of similar diameter and thickness are also given.

\begin{tabular}{|c|c|c|c|c|c|c|}
\hline$t(\mathrm{~nm}) / d(\mathrm{~nm})$ & $\alpha_{n}\left(10^{-4} / \mathrm{K}^{3 / 2}\right)$ & $\alpha_{a n}\left(10^{-4} / \mathrm{K}^{3 / 2}\right)$ & $\beta_{n}\left(10^{-2} / \mathrm{K}\right)$ & $\beta_{a n}\left(10^{-3} / \mathrm{K}\right)$ & $H_{n}^{r t}(\mathrm{Oe})$ & $H_{a n}^{r t}(\mathrm{Oe})$ \\
\hline \multicolumn{7}{|c|}{ Closely packed Py caps on $\mathrm{SiO}_{2}$ particles } \\
\hline $20 / 330$ & $14.0 \pm 1.0$ & $8.8 \pm 0.2$ & $1.2 \pm 0.1$ & $16.0 \pm 1.2$ & $-33 \pm 10$ & $-166 \pm 10$ \\
\hline $40 / 330$ & $90.0 \pm 2.0$ & $6.9 \pm 0.4$ & $1.7 \pm 0.2$ & $5.0 \pm 0.4$ & $+13 \pm 5$ & $-204 \pm 10$ \\
\hline $40 / 800$ & $1.3 \pm 0.1$ & $7.0 \pm 0.2$ & $2.0 \pm 0.3$ & $4.5 \pm 0.7$ & $+38 \pm 5$ & $-185 \pm 10$ \\
\hline \multicolumn{7}{|c|}{ Individual planar Py disks (Ref. 22) } \\
\hline $50 / 526$ & $0.08 \pm 0.02$ & $1.0 \pm 0.2$ & $0.8 \pm 0.1$ & $1.3 \pm 0.2$ & $+470 \pm 10$ & $-940 \pm 20$ \\
\hline $50 / 865$ & $0.1 \pm 0.03$ & $0.3 \pm 0.05$ & $1.1 \pm 0.4$ & $5.9 \pm 1.9$ & $+290 \pm 15$ & $-620 \pm 20$ \\
\hline
\end{tabular}

smaller magnetostatic intercap coupling compared to planar counterparts. Closely packed caps with a diameter of $330 \mathrm{~nm}$ revealed a magnetic frustrated state that we called the chirality frustrated vortex state. As the name suggests, these states are frustrated by the sense of spin curling instead of a moment frustration reported in literature. The large separation in arrays of caps with $800 \mathrm{~nm}$ diameter suppresses such kind of coupling. We further investigated the magnetic coupling by determining the stability of the vortex state in cap structures and gave a comparison to their planar counterparts.

\section{ACKNOWLEDGMENTS}

The authors thank I. Fiering for sputter deposition, A. Wolter-Giraud for offering SQUID-VSM measurements, and D. J. Thurmer for SEM imaging (IFW Dresden); V. Neu and R. Schäfer (IFW Dresden), D. D. Sheka (NTSU Kiev), Y. Gaididei (BITP Kiev), and S. Thomas (TU Chemnitz) are acknowledged for fruitful discussions. This work was financed via the German Science Foundation (DFG) Grants No. MA 5144/1-1, MA 5144/2-1 and DFG Research Unit 1713. *d.makarov@ifw-dresden.de

${ }^{1}$ R. P. Cowburn, D. K. Koltsov, A. O. Adeyeye, M. E. Welland, and D. M. Tricker, Phys. Rev. Lett. 83, 1042 (1999).

${ }^{2}$ A. Wachowiak, J. Wiebe, M. Bode, O. Pietzsch, M. Morgenstern, and R. Wiesendanger, Science 298, 577 (2002).

${ }^{3}$ S.-B. Choe, Y. Acremann, A. Schollm, A. Bauer, A. Doran, J. Stöhr, and H. A. Padmore, Science 304, 420 (2004).

${ }^{4}$ B. Van Waeyenberge, A. Puzic, H. Stoll, K. W. Chou, T. Tyliszczak, M. Fähnle, H. Brückl, K. Rott, G. Reiss, I. Neudecker, D. Weiss, C. H. Back, and G. Schütz, Nature (London) 444, 461 (2006).

${ }^{5}$ A. Vansteenkiste, K. W. Chuo, M. Weigand, M. Curcic, V. Sackmann, H. Stoll, T. Tyliszczak, G. Woltersdorf, C. H. Back, G. Schütz, and B. Van Waeyenberge, Nat. Phys. 5, 332 (2009).

${ }^{6}$ M. Kammerer, M. Weigand, M. Curcic, M. Noske, M. Sproll, A. Vansteenkiste, B. VanWaeyenberge, H. Stoll, G. Woltersdorf, C. H. Back, and G. Schütz, Nat. Commun. 2, 279 (2011).

${ }^{7}$ K. Yamada, S. Kasai, Y. Nakatani, K. Kobayashi, H. Kohno, A. Thiaville, and T. Ono, Nat. Mater. 6, 269 (2007).

${ }^{8}$ V. Kravchuk, D. Sheka, Y. Gaididei, and F. Mertens, J. Appl. Phys. 102, 043908 (2007).

${ }^{9}$ J.-G. Zhu, Y. Zheng, and G. A. Prinz, J. Appl. Phys. 87, 6668 (2000).

${ }^{10}$ K. Y. Guslienko, V. Novosad, Y. Otani, H. Shima, and K. Fukamichi, Phys. Rev. B 65, 024414 (2001).

${ }^{11}$ N. Martin, N.-C. Bigall, I. Mönch, T. Gemming, A. Eychmüller, R. Mattheis, R. Schäfer, L. Schultz, and J. McCord, Adv. Funct. Mater. 21, 891 (2011).

${ }^{12}$ M. Albrecht, G. Hu, I. L. Guhr, T. C. Ulbrich, J. Boneberg, P. Leiderer, and G. Schatz, Nat. Phys. 4, 203 (2005).

${ }^{13}$ T. C. Ulbrich, D. Makarov, G. Hu, I. L. Guhr, D. Suess, T. Schre, and M. Albrecht, Phys. Rev. Lett. 96, 077202 (2006).
${ }^{14}$ D. Makarov, L. Baraban, I. L. Guhr, J. Boneberg, H. Schift, J. Gobrecht, G. Schatz, P. Leiderer, and M. Albrecht, Appl. Phys. Lett. 90, 093117 (2007).

${ }^{15}$ V. P. Kravchuk, D. D. Sheka, R. Streubel, D. Makarov, O. G. Schmidt, and Y. Gaididei, Phys. Rev. B 85, 144433 (2012).

${ }^{16}$ R. F. Wang, C. Nisoli, R. S. Freitas, J. Li, W. McConville, B. J. Cooley, M. S. Lund, N. Samarth, C. Leighton, V. H. Crespi, and P. Schiffer, Nature (London) 439, 303 (2006).

${ }^{17}$ E. Mengotti, L. Heyderman, A. Rodriguez, F. Nolting, R. Hugli, and H.-B. Braun, Nat. Phys. 7, 68 (2011).

${ }^{18} \mathrm{We}$ used the OOMMF code, version $1.2 \mathrm{a} 4$ [http://math.nist. gov/oommf/]. All simulations were performed for material parameters of permalloy: exchange constant $A=1.3 \times 10^{-11} \mathrm{~J} / \mathrm{m}$; saturation magnetization $M_{s}=8.6 \times 10^{5} \mathrm{~A} / \mathrm{m}$; and the anisotropy was neglected. The mesh size was $2 \mathrm{~nm}$.

${ }^{19}$ C. M. Schneider and G. Schönhense, Rep. Prog. Phys. 65, R1785 (2002).

${ }^{20}$ J. Kimling, F. Kronast, S. Martens, T. Böhnert, M. Martens, J. Herrero-Albillos, L. Tati-Bismaths, U. Merkt, K. Nielsch, and G. Meier, Phys. Rev. B 84, 174406 (2011).

${ }^{21}$ M. V. Sapozhnikov, O. L. Ermolaeva, B. G. Gribkov, I. M. Nefedov, I. R. Karetnikova, S. A. Gusev, V. V. Rogov, B. B. Troitskii, and L. V. Khokhlova, Phys. Rev. B 85, 054402 (2012).

${ }^{22}$ G. Mihajlovic, M. S. Patrick, J. E. Pearson, V. Novosad, S. D. Bader, M. Field, G. J. Sullivan, and A. Hoffmann, Appl. Phys. Lett. 96, 112501 (2010)

${ }^{23}$ K. Maaz, A. Mumtaz, S. K. Hasanain, and M. F. Bertino, J. Magn. Magn. Mater. 322, 2199 (2010).

${ }^{24}$ M. P. Sharrock, J. Appl. Phys. 76, 6413 (1994).

${ }^{25}$ P. Gaunt, J. Appl. Phys. 59, 4129 (1986). 